\title{
Effect of eribulin on patients with metastatic breast cancer: multicenter retrospective observational study in Taiwan
}

\author{
Kun-Ming Rau ${ }^{1,12} \cdot$ Fu Ou-Yang ${ }^{2,12,13} \cdot$ Ta-Chung Chao $^{3} \cdot$ Yao-Lung Kuo $^{4} \cdot$ Tsui-Fen Cheng $^{5} \cdot$ Tsu-Yi Chao $^{6}$.

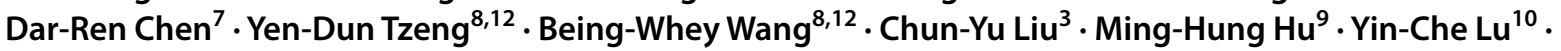 \\ Wei-Jen Ou ${ }^{11}$. Chin-Ho Kuo ${ }^{10}$. Chieh-Han Chuang ${ }^{2,12,13}$ • Jung-Yu Kan ${ }^{2,12,13}$ • Fang-Ming Chen 2,12,13,14,15 . \\ Ming-Feng $\mathrm{Hou}^{2,12,13,15,16}$ (B)
}

Received: 29 March 2018 / Accepted: 30 March 2018 / Published online: 5 April 2018

(c) The Author(s) 2018

\begin{abstract}
Purpose The aim of this study was to confirm the therapeutic role of eribulin on Taiwanese women with metastatic breast cancer.

Methods This retrospective study examined 449 females who received eribulin between March 2014 and June 2017 at 14 hospitals in Taiwan for treatment of locally advanced or metastatic breast cancer.

Results The survival rate at 24 months was $57.2 \%$ (95\% CI 51.0-62.9\%) and the median time to treatment failure (TTF) was 3.91 months (95\% CI 3.45-3.94). A total of 175 patients (40.1\%) received eribulin for fewer than 90 days and the others received it for 90 days or more. Eight patients (1.83\%) had complete remission, 82 (18.8\%) had partial remission, 202 (46.3\%) had stable disease, and 144 (33.0\%) had progressive disease (PD). Patients' tumors with the luminal A subtype had a significantly better objective response rate. Kaplan-Meier analysis indicated that hormone receptor positivity, luminal A subtype, receipt of eribulin as the 1st to 3rd line therapy, and metastasis to fewer than 4 organs were significantly associated with longer TTF. Stepwise multivariate analysis showed that only receipt of eribulin as the 1st to 3rd line therapy was significantly associated with TTF (HR 1.49, $p<0.001)$. All toxicities were manageable and only 18 patients $(4.1 \%)$ discontinued treatment due to adverse events.

Conclusions Eribulin appears to have better efficacy and cause fewer adverse events, especially neutropenia, in Taiwanese women than Western women.
\end{abstract}

Keywords Eribulin $\cdot$ Metastatic breast cancer $\cdot$ Efficacy $\cdot$ Safety $\cdot$ Real world $\cdot$ Taiwanese women

\section{Introduction}

Breast cancer is the most common cancer among women worldwide, and approximately $10 \%$ of newly diagnosed cases are stage IV (metastatic) [1]. Several treatments are available for patients with metastatic breast cancer (MBC), but none of them are curative treatments, so these patients

Kun-Ming Rau, Fu Ou-Yang and Ta-Chung Chao have contributed equally to this work.

Fang-Ming Chen

fchen@kmu.edu.tw

Ming-Feng Hou

mifeho@kmu.edu.tw

Extended author information available on the last page of the article tend to have poor long-term survival rates, with median survival times of 18-24 months [2]. The goals of most treatments of these patients are palliation and improvement in the quality of life. Taxanes and anthracyclines are the standard adjuvant and first-line treatments for women with MBC, but these treatments are not always successful due to the development of drug resistance [3]. Thus, it is difficult to treat patients after failure of these therapies, and there are no established regimens for subsequent treatment.

Microtubules have an important function in the mitosis of normal and cancer cells. Thus, several agents that target microtubules are used to treat different cancers. These include paclitaxel and docetaxel, which prevent microtubule depolymerization, and vincristine and vinblastine, which inhibit microtubule formation [4]. These drugs disrupt 
normal function of microtubule during mitosis, leading to cell death.

Eribulin (MW: 826.0, $\mathrm{C}_{40} \mathrm{H}_{59} \mathrm{NO}_{11} \cdot \mathrm{CH}_{4} \mathrm{O}_{3} \mathrm{~S}$ ) is an antitubulin agent that was first isolated from a marine sponge [5]. Studies of its mechanism of action indicated that, in contrast to other microtubule-targeting agents, eribulin prevents the formation of cross-links between different sulfhydryl groups in $\beta$-tubulin [6]. Thus, eribulin inhibits microtubule growth, leading to an accumulation of non-functional aggregates of tubulin, and then mitosis arrests at metaphase/ anaphase. In addition, studies of several lines of cancer cells indicated that eribulin has additive or synergistic effects with other antineoplastic agents [6].

Eribulin was initially approved in 2010 for treatment of MBC in patients who previously completed at least 2 chemotherapy regimens [7]. Although it is now approved for this indication in many countries, there are limited studies of its effect in Asian women. There was a study of the efficacy of eribulin in 80 Japanese women with MBC [8], but most previous studies only included $\sim 2 \%$ Asian women [9-12]. Several clinical trials had proved the effect and safety of eribulin on locally advanced breast cancer (LABC) or MBC [6], but the real-world experiences of eribulin treatment were still few to be reported, especially for patients who do not fit the criteria of clinical trials. The benefits and toxicities of eribulin may differ among Western and Asian women. Here we performed a multicenter, retrospective investigation to determine the effect of eribulin in Taiwanese patients with MBC. Because some doctors would choose either capecitabine or vinorelbine as a salvage or maintenance therapy for advanced breast cancer after anthracycline or taxane, here we also would check the impact of previous exposure to these two drugs on eribulin treatment.

\section{Methods}

\section{Patients}

The clinical data of patients who received eribulin between March 2014 and June 2017 at 14 hospitals in Taiwan were retrospectively collected and reviewed (Clinical Trial Registry Number: NCT03245112). Included patients should be with pathological confirmed LABC or MBC, and had been treated by an anthracycline and a taxane regimen before, either as the adjuvant or metastatic purpose. There were no exclusions based on age or menopausal status, and none of the patients should be pregnant or nursing. The characteristics of tumors such as estrogen receptor (ER), progesterone receptor $(\mathrm{PgR})$, human epidermal growth factor receptor 2 (Her-2), Ki-67, and molecular subtypes (luminal A, luminal B, Her-2 enriched, and triple-negative breast cancer
[TNBC]) were recorded. Metastatic sites including lymph nodes and distant organs were also recorded and analyzed.

\section{Treatment}

The treatment consisted of monotherapy with intravenous eribulin (over 2-5 min), which was administered at a dose of $1.4 \mathrm{mg} / \mathrm{m}^{2}$ on days 1 and 8 of a 21 -day cycle. The reported data included characteristics of tumors, clinical parameters (e.g., site of metastases), treatment events (e.g., number of therapeutic cycles, start/end dates, and rationale for discontinuation), clinical response, use of supportive care medications (e.g., granulocytic colony stimulating factor), dose adjustment, and adverse events. The reported results are based on effectiveness analysis of data collected by June 2017.

\section{Outcome measures}

The primary outcome measure is disease control rate (DCR), which was defined as proportions of patients who achieved complete response (CR), partial response (PR), and stable disease (SD) as the best response. Thus, the percentage of patients with $\mathrm{LABC}$ or MBC who achieved CR, PR, and $\mathrm{SD}$ were recorded during eribulin treatment. The secondary outcome measure is the safety of eribulin, i.e., the number of patients with adverse events (AE) and severity of AE which were assessed using the Common Terminology Criteria for Adverse Events (CTCAE), version 4.0. This includes all events that were not present before the initial administration of eribulin, pre-existing events that became more intense or more frequent, and events that were present upon initial eribulin administration, but became more severe following administration.

\section{Statistical analysis}

Characteristics of patients and tumors, treatment duration, tumor response, and other categorical variables are summarized $n(\%)$, and age as median (range). The overall survival (OS) time was defined as the time from treatment onset to death or the last follow-up, which will be presented as mean with $95 \%$ confidence interval (CI). The time to treatment failure (TTF) was defined as the period from the first dose of eribulin to cancelation for any reason (including death, disease aggravation, treatment toxicity, or patient's request), or was censored at the date of last follow-up for surviving patients remaining on treatment which will be presented as mean with $95 \%$ CI. Univariate analysis was performed to determine the association of tumor responses (objective response rate [ORR], CR + PR; and DCR, CR + PR + SD) with patient characteristics. The differences of tumor responses were compared using 
one-way ANOVA for age, and the Pearson Chi-square $\left(\chi^{2}\right)$ test or Fisher's exact test for other categorical variables. Univariate and multivariate binary logistic regression were also applied to determine the association between tumor responses and patient characteristics and presented as odds ratios (ORs), 95\% CIs, and $p$ values. Kaplan-Meier $(\mathrm{K}-\mathrm{M})$ curves for overall survival (OS) and TTF were also determined. Furthermore, the $\mathrm{K}-\mathrm{M}$ curve and logrank test were used to assess the association of TTF with different characteristics. In particular, the median, 95\% $\mathrm{CI}$, and $p$ value of TTF for each characteristic were calculated using the log-rank test. Univariable Cox-regression analyses were also used to identify the association of TTF and different characteristics, and a stepwise multivariable Cox-regression analysis was used to analyze variables with $p$ values below 0.2 in the univariable analysis. All statistical assessments were two-tailed and a $p$ value below 0.05 was considered significant. All data analysis used Stata Statistical Software (Release 11, StataCorp LP, College Station, TX).

\section{Results}

\section{Characteristics}

The medical records of a total of 449 patients from 14 hospitals who received eribulin were retrospectively reviewed. Six patients were excluded because of loss to follow-up. Thus, we initially recruited 443 patients who were scheduled for treatment. Among these patients, 436 patients received at least one dose of eribulin and were used to assess efficacy, and 440 patients were used to assess safety.

The median age was 51.6 years old (range 22.2-81.0), $27.8 \%$ of patients were Her-2 positive, $64.9 \%$ were ER positive, and $49.1 \%$ were $\mathrm{PgR}$ positive. Analysis of the molecular subtypes indicated that $222(50.9 \%)$ patients were luminal A, 68 (15.6\%) were luminal B, 51 (11.7\%) were Her-2 enriched, and 75 (17.2\%) were TNBC. The median number of previous chemotherapy regimen was 3 (0-12). A total of 207 patients $(47.5 \%)$ received eribulin as a 4th line or later treatment, 215 patients $(49.3 \%)$ received eribulin as a 1 st to 3 rd line treatment, and the line of treatment was unknown for 14 patients (3.21\%). There were 221 patients $(50.7 \%)$ who received previous capecitabine and 217 patients $(47.8 \%)$ who received previous vinorelbine; 142 (32.6\%) of patients received neither of these agents, and 144 (33.0\%) of patients received both agents. Most patients had metastasis to a single site $(n=224,51.4 \%)$; bone $(n=157,36.0 \%)$, lung $(n=141$, $32.3 \%)$, and liver $(n=118,27.1 \%)$ were the most common sites of metastases. (Table 1 ).
Table 1 Clinical characteristics of enrolled patients. $(n=436)$

\begin{tabular}{|c|c|c|}
\hline Characteristic & $n$ & $\%$ \\
\hline \multicolumn{3}{|l|}{ ER } \\
\hline Positive & 283 & 64.91 \\
\hline Negative & 139 & 31.88 \\
\hline Unknown & 14 & 3.21 \\
\hline \multicolumn{3}{|l|}{ PR } \\
\hline Positive & 214 & 49.08 \\
\hline Negative & 207 & 47.48 \\
\hline Unknown & 15 & 3.44 \\
\hline \multicolumn{3}{|l|}{ Her-2 } \\
\hline Positive & 121 & 27.75 \\
\hline Negative & 300 & 68.81 \\
\hline Unknown & 15 & 3.44 \\
\hline \multicolumn{3}{|l|}{ Molecular subtype } \\
\hline Luminal A & 222 & 50.92 \\
\hline Luminal B & 68 & 15.60 \\
\hline Her-2 enriched & 51 & 11.70 \\
\hline TNBC & 75 & 17.20 \\
\hline Unknown & 20 & 4.59 \\
\hline \multicolumn{3}{|l|}{ Previous chemotherapy } \\
\hline Capecitabine & 221 & 50.69 \\
\hline Vinorelbine & 217 & 49.77 \\
\hline Capecitabine alone & 77 & 17.66 \\
\hline Vinorelbine alone & 73 & 16.74 \\
\hline Capecitabine \& Vinorelbine naïve & 142 & 32.57 \\
\hline Capecitabine \& Vinorelbine & 144 & 33.03 \\
\hline \multicolumn{3}{|l|}{ Therapy line of eribulin } \\
\hline 1 & 59 & 13.53 \\
\hline 2 & 67 & 15.37 \\
\hline 3 & 89 & 20.41 \\
\hline$>3$ & 207 & 47.48 \\
\hline Unknown & 14 & 3.21 \\
\hline \multicolumn{3}{|l|}{ Sites with metastasis } \\
\hline 1 & 224 & 51.38 \\
\hline 2 & 84 & 19.27 \\
\hline 3 & 75 & 17.20 \\
\hline$\geq 4$ & 53 & 12.16 \\
\hline \multicolumn{3}{|l|}{ Organs with metastasis } \\
\hline Bone & 157 & 36.01 \\
\hline Lung & 141 & 32.34 \\
\hline $\mathrm{LN}$ & 81 & 18.58 \\
\hline Liver & 118 & 27.06 \\
\hline Brain & 53 & 12.16 \\
\hline Skin & 28 & 6.42 \\
\hline
\end{tabular}

$E R$ estrogen receptor, Her2 human epidermal growth factor receptor 2, $P R$ progesterone receptor, $L N$ lymph node, $T N B C$ triple-negative breast cancer 


\section{Efficacy of treatment}

As of Nov. 30, 2017, 142 patients (32.6\%) had expired, so the median OS time could not be calculated. The OS rate at 24 months was $57.2 \%$ (95\% CI 51.0-62.9\%, Fig. 1a) and the median TTF was 3.91 months (95\% CI 3.45-3.94, Fig. 1b). Stratify the patients by the treatment duration, the results indicated that 175 patients $(40.1 \%)$ received eribulin for fewer than 90 days and the others received eribulin for 90 days or more. The median cycles of eribulin administration were 4.

Analysis of tumor response indicated that 8 patients (1.83\%) were CR, 82 (18.8\%) were PR, 202 (46.3\%) were SD, and 144 (33.0\%) were PD (Table 2). The ORR was $20.6 \%$ and the DCR was $67.0 \%$. The primary reasons for treatment discontinuation were disease progression (88.6\%), adverse events (4.1\%), patient's request (3.7\%), and death (1.9\%) (data not shown).

Different subtypes also had different responses to eribulin. In general, luminal A subtype had significantly better ORR (25.7\%) than those with the luminal B (17.7\%), Her-2 enriched (21.6\%), TNBC (9.33\%), and unknown
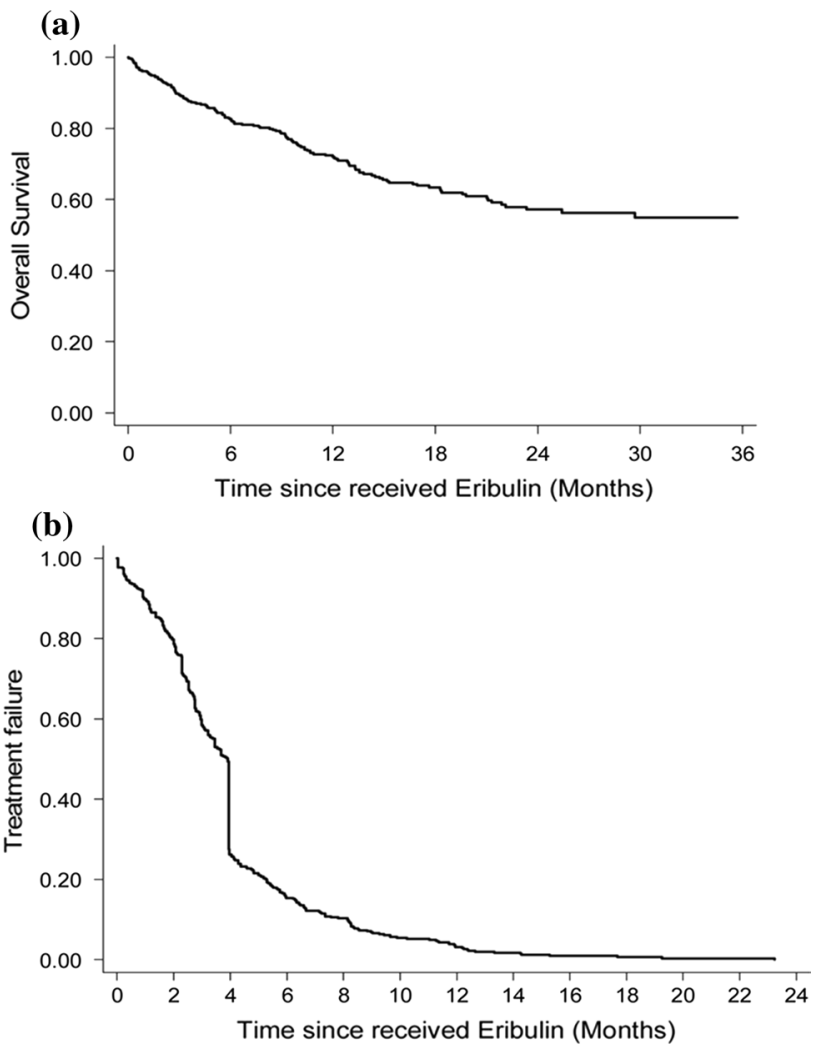

Fig. 1 Kaplan-Meier curves of $\mathbf{a}$ overall survival and $\mathbf{b}$ time to treatment failure. The mean OS was 13.4 months (95\% CI 0.26-35.06) and the median TTF was 3.91 months (95\% CI 3.45-3.94 months). Abbreviations: $O S$ overall survival, $T T F$ time to treatment failure. $C I$ confidence interval, $N A$ not assessed
Table 2 Treatment and tumor responses

\begin{tabular}{lll}
\hline Characteristic & $n(\%)$ or as indicated \\
\hline $\begin{array}{l}\text { Eribulin treatment duration } \\
\quad<90 \text { days }\end{array}$ & 175 & $(40.14 \%)$ \\
$\quad \geq 90$ days & 261 & $(59.86 \%)$ \\
TTF, months & & \\
$\quad$ Mean \pm SD & 3.99 & $( \pm 3.06)$ \\
Median (range) & 3.91 & $(0.03-23.23)$ \\
Response & & \\
CR & 8 & $(1.83 \%)$ \\
PR & 82 & $(18.81 \%)$ \\
SD & 202 & $(46.33 \%)$ \\
PD & 144 & $(33.03 \%)$ \\
\hline
\end{tabular}

$C R$ complete response, $P D$ progressive disease, $P R$ partial response, $S D$ stable disease

$(15.0 \%)$ subtypes $(p=0.032)$. Moreover, univariate and multivariate analysis of factors associated with ORR indicated that patients with the TNBC subtype had a lower ORR than those with luminal A subtype (OR 0.30, 95\% CI $0.13-0.69, p=0.004)$, this association remained in the multivariable analysis (OR $0.18,95 \%$ CI $0.04-0.77$, $p=0.021$ ) (data not shown). We also show that ORR was higher in patients who were prior capecitabine and vinorelbine naïve $(25.4 \%)$, received eribulin as the first line $(28.8 \%)$ or second to third line $(21.2 \%)$, and had metastasis to fewer than 4 sites (88.9\%), although none of these differences were statistically significant (Table 3).

We used K-M survival analysis and the log-rank test to assess the relationship of different demographic and clinical characteristics with TTF. These results show that luminal A subtype, receipt of eribulin as the 1st, 2nd, or 3rd line, ORR, and metastasis to fewer than four organs were significantly associated with longer TTF ( $p<0.05$ for all comparisons) (Fig. 2).

We also performed univariate and multivariate analysis of the relationship of TTF with different demographic and clinical characteristics. The univariate results showed that TNBC subtype (HR 1.65, $p<0.001$ ), previous treatment with capecitabine and vinorelbine (HR 1.28, $p=0.042$ ), and metastasis to 4 sites or more (HR 1.40, $p=0.024$ ) were significantly associated with worse TTF. The stepwise multivariate analysis, which considered variables whose $p$ values were less than 0.2 in the univariate analysis, showed that only receipt of eribulin as the 1 st, $2 \mathrm{nd}$, or 3rd line therapy was significantly associated with better TTF (HR 1.49, $p<0.001$ ) (Table 4). 
Table 3 Relationship of tumor characteristics with response

\begin{tabular}{|c|c|c|c|c|c|c|c|}
\hline \multirow[t]{2}{*}{ Characteristics } & \multicolumn{2}{|c|}{$\mathrm{CR}+\mathrm{PR}$} & \multicolumn{2}{|l|}{ SD } & \multicolumn{2}{|l|}{ PD } & \multirow[t]{2}{*}{$P$} \\
\hline & $n$ & $\%$ & $n$ & $\%$ & $n$ & $\%$ & \\
\hline Total cases & 90 & 20.64 & 202 & 46.33 & 144 & 33.03 & \\
\hline Molecular subtype & & & & & & & $0.032^{\mathrm{b}}$ \\
\hline Luminal A & 57 & 25.68 & 98 & 44.14 & 67 & 30.18 & \\
\hline Luminal B & 12 & 17.65 & 37 & 54.41 & 19 & 27.94 & \\
\hline Her-2 enriched & 11 & 21.57 & 21 & 41.18 & 19 & 37.25 & \\
\hline TNBC & 7 & 9.33 & 34 & 45.33 & 34 & 45.33 & \\
\hline Unknown & 3 & 15.00 & 12 & 60.00 & 5 & 25.00 & \\
\hline \multicolumn{8}{|l|}{ Previous chemotherapy regimen } \\
\hline Capecitabine & 41 & 18.55 & 102 & 46.15 & 78 & 35.29 & $0.439^{\mathrm{b}}$ \\
\hline Vinorelbine & 40 & 18.43 & 98 & 45.16 & 79 & 36.41 & $0.267^{\mathrm{b}}$ \\
\hline Capecitabine alone & 14 & 18.18 & 35 & 45.45 & 28 & 36.36 & $0.406^{\mathrm{b}}$ \\
\hline Vinorelbine alone & 13 & 17.81 & 31 & 42.47 & 29 & 39.73 & \\
\hline Capecitabine \& Vinorelbine naïve & 36 & 25.35 & 69 & 48.59 & 37 & 26.06 & \\
\hline Capecitabine \& Vinorelbine & 27 & 18.75 & 67 & 46.53 & 50 & 34.72 & \\
\hline Eribulin therapy line after metastases & & & & & & & $0.210^{\mathrm{b}}$ \\
\hline 1 & 17 & 28.81 & 29 & 49.15 & 13 & 22.03 & \\
\hline $2-3$ & 33 & 21.15 & 65 & 41.67 & 58 & 37.18 & \\
\hline$>3$ & 39 & 18.84 & 96 & 46.38 & 72 & 34.78 & \\
\hline Unknown & 1 & 7.14 & 12 & 85.71 & 1 & 7.14 & \\
\hline Metastatic sites & & & & & & & $0.392^{\mathrm{b}}$ \\
\hline$<4$ & 80 & 88.89 & 181 & 89.60 & 122 & 84.72 & \\
\hline$\geqq 4$ & 10 & 11.11 & 21 & 10.40 & 22 & 15.28 & \\
\hline
\end{tabular}

${ }^{\mathrm{a}} p$ value estimated by one-way ANOVA test

${ }^{\mathrm{b}} p$ value estimated by one-way Chi-squared test

\section{Adverse events}

We assessed the safety of eribulin by analysis of 440 patients, there were 18 patients $(4.1 \%)$ who discontinued treatment due to AEs. Neutropenia (21.3\%) and leukopenia $(16.7 \%)$ were the major hematological adverse events. The most common non-hematological AEs were alopecia (26.4\%), fatigue or lethargy (21.3\%), and nausea (11.5\%). A total of 86 patients $(19.7 \%)$ received treatment with granulocyte colony stimulating factor for chemotherapy-induced neutropenia (Table 5).

\section{Discussion}

Several clinical studies of eribulin evaluating its safety and efficacy in real-world clinical settings had been published previously. For example, in Watanabe et al. [13], they reported a post-marketing observational study in Japanese patients with locally advanced or metastatic breast cancer, of these, 671 patients were included in the effectiveness analysis. They found that CR and PR were observed in 1.3 and $15.2 \%$ of patients, respectively. The ORR was $16.5 \%$, DCR was $50.1 \%$, clinical benefit rate was $22.4 \%$, and the median TTF was 127 days. On the other hand, Garrone et al. performed a multicenter study of the effect of eribulin on 113 Italian women who were previously treated for MBC to assess its effect in an actual clinical setting [14]. They reported an ORR of $24 \%$, and a clinical benefit rate of $35.4 \%$. Moreover, after a median follow-up time of 29.6 months, the median PFS was 3.3 months, and the median OS was 11.6 months. They concluded that eribulin was safe and effective in a real-world clinical setting, in agreement with our results. Watanabe [15] also studied eribulin in a real-world clinical setting. In particular, he retrospectively examined the effect of eribulin monotherapy on survival of Japanese women with ER-positive and Her-2-negative MBC. Sixty-six women received eribulin and 227 received a conventional chemotherapy agent. The results indicated significantly better OS in patients receiving eribulin. Moreover, the survival benefit did not depend on which organs had metastases or the use of previous chemotherapy regimens. In agreement with the results of Watanabe [15], we found that all 7 of our patients who had CRs were Her-2 negative and ER positive, and 6 of them were PgR positive. Gamucci et al. [16] retrospectively studied 133 Italian women with advanced/metastatic breast cancer who previously received 2 or more lines of chemotherapy. They reported that the ORR 

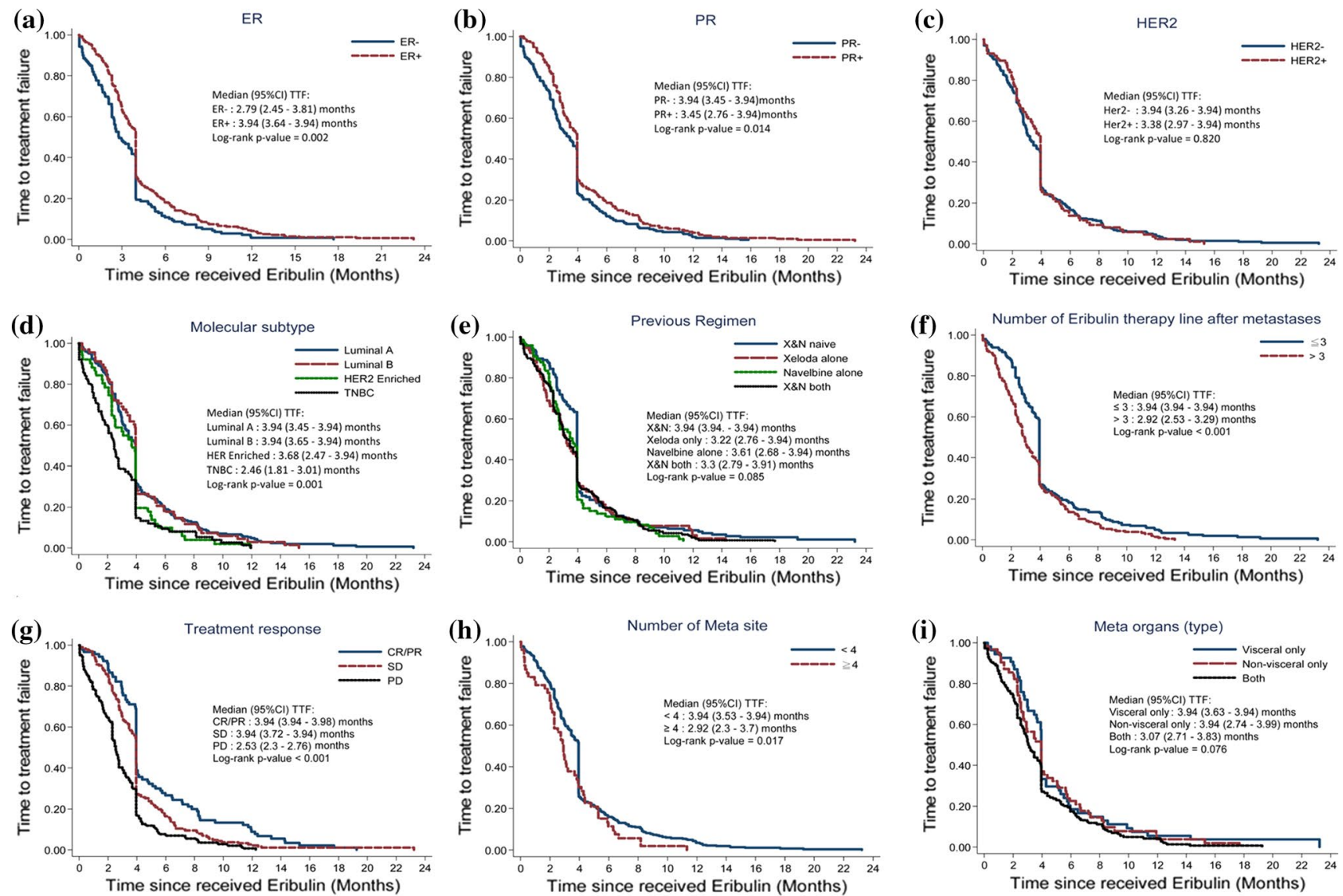

Fig. 2 Kaplan-Meier curves of time to treatment failure (TTF) according to a ER status, b PR status, $\mathbf{c}$ Her-2 status, d molecular subtype, e previous regimen, $\mathbf{f}$ therapy line of eribulin, $\mathbf{g}$ treatment

was $21.1 \%$, SD in $42.8 \%$ of patients, and the presence of PR or SD for at least 6 months in $38.3 \%$ of patients. They also found that eribulin was especially effective when given to women with Her-2-negative tumors.

There are several important results of the present retrospective study of the effect of eribulin on Taiwanese women with $\mathrm{MBC}$ or $\mathrm{LABC}$. Similar to the results of a previous study of Japanese women [15], Taiwanese women who had hormone receptor-positive and Her-2-negative $\mathrm{BC}$ had better outcomes than those who were Her-2 positive. Although the pooled analysis of trial 301 and EMBRACE study reported that eribulin was more effective for TNBC, the researchers only compared it with the control arm [11]. Real-world data indicate that luminal A patients had better therapeutic outcomes than those who were Her-2 positive or with TNBC. In addition, TTF in the current study was consistent with the prior study [13]. In the current study, patients also had better overall survival time than reported in another study in a real other world setting [17]. This might be because the availability of more treatment options in recent years that extended the survival time of our patients. response, $\mathbf{h}$ number of metastatic sites, $\mathbf{i}$ metastatic organs. Medians and $95 \%$ confidence intervals (95\% CIs) were calculated using the log-rank test

There was greater variation in the number of lines on previous therapy before eribulin treatment for patients in the present study than in most previous studies. Our patient population was more like those in the EMBRACE study, and our results are at least non-inferior to those in the EMBRACE study [9]. In particular, we examined patients who received 1 prior regimen to more than 3 prior regimens (median: 4), we found that ORR was better for patients who received fewer prior treatments, and K-M analysis indicated a small increase in TTF of patients who received 3 or fewer lines of therapy, relative to those who received 4 or more lines (3.94 months vs. 2.92 months, $p<0.001$, Fig. 2f), although DCR was not significantly different for those with different numbers of prior treatments. In addition, response rate in the current study was higher than that of previous phase II [18], phase IV [19], and retrospective studies [13] among Asian patient population. This suggests that eribulin could be considered for patients with a wide variety of treatment histories, and it is reasonable that earlier use of eribulin provides a better ORR. 
Table 4 Univariate and multivariate Cox proportional hazard regression analysis of factors associated with time to treatment failure (TTF). $(n=436)$

\begin{tabular}{|c|c|c|c|c|c|c|c|c|c|}
\hline \multirow[t]{2}{*}{ Variables } & \multicolumn{3}{|c|}{ Univariate } & \multicolumn{3}{|c|}{ Multivariate $^{\mathrm{a}}$} & \multicolumn{3}{|c|}{ Multivariate $^{\mathrm{b}}$} \\
\hline & HR & $95 \% \mathrm{CI}$ & $P$ & HR & $95 \% \mathrm{CI}$ & $P$ & HR & $95 \% \mathrm{CI}$ & $P$ \\
\hline \multicolumn{10}{|l|}{ Molecular subtype } \\
\hline Luminal A & 1.00 & & & 1.00 & & & 1.00 & & \\
\hline Luminal B & 0.99 & $0.76-1.31$ & 0.965 & 1.08 & $0.6-1.93$ & 0.796 & 0.95 & $0.72-1.26$ & 0.729 \\
\hline Her-2 enriched & 1.32 & $0.97-1.79$ & 0.077 & 1.78 & $0.73-4.33$ & 0.205 & 1.50 & $0.76-2.97$ & 0.241 \\
\hline TNBC & 1.65 & $1.26-2.14$ & $<0.001$ & 1.92 & $0.96-3.83$ & 0.066 & 1.79 & $0.93-3.47$ & 0.083 \\
\hline Previous capecitabine & 1.16 & $0.96-1.4$ & 0.126 & - & & & - & & \\
\hline Previous vinorelbine & 1.20 & $0.99-1.45$ & 0.061 & - & & & - & & \\
\hline \multicolumn{10}{|l|}{ Previous regimen } \\
\hline Capecitabine \& vinorelbine naïve & 1.00 & & & 1.00 & & & 1.00 & & \\
\hline Capecitabine alone & 1.25 & $0.95-1.66$ & 0.115 & 1.00 & $0.73-1.37$ & 0.994 & 1.11 & $0.82-1.5$ & 0.504 \\
\hline Vinorelbine alone & 1.33 & $1-1.77$ & 0.052 & 1.06 & $0.76-1.47$ & 0.721 & 1.04 & $0.76-1.41$ & 0.821 \\
\hline Capecitabine \& vinorelbine & 1.28 & $1.01-1.61$ & 0.042 & 0.94 & $0.7-1.26$ & 0.663 & 1.01 & $0.77-1.32$ & 0.960 \\
\hline \multicolumn{10}{|l|}{ Eribulin therapy line after metastases } \\
\hline$\leqq 3$ & 1.00 & & & 1.00 & & & 1.00 & & \\
\hline$>3$ & 1.25 & $0.95-1.66$ & 0.115 & 1.47 & $1.16-1.86$ & $<0.001$ & 1.49 & $1.19-1.86$ & $<0.001$ \\
\hline \multicolumn{10}{|l|}{ Meta site no. } \\
\hline$<4$ & 1.00 & & & 1.00 & & & 1.00 & & \\
\hline$\geqq 4$ & 1.40 & $1.04-1.86$ & 0.024 & 1.31 & $0.85-2.02$ & 0.226 & 1.11 & $0.82-1.51$ & 0.489 \\
\hline Organs with metastasis ${ }^{c}$ & & & & - & & & - & & \\
\hline Visceral only & Ref & & & & & & & & \\
\hline Non-visceral only & 1.12 & $0.77-1.62$ & 0.548 & & & & & & \\
\hline Both & 1.38 & $1-1.9$ & 0.048 & & & & & & \\
\hline
\end{tabular}

1.00: Reference category

${ }^{a}$ Included all variables in univariate analysis

${ }^{\mathrm{b}}$ Included variables with $p$ value less than 0.2 from univariate analysis

${ }^{\mathrm{c}}$ Unknown meta organ type was excluded $(n=176)$

Table 5 Adverse events. $(n=440)$

\begin{tabular}{lllll}
\hline & All grades & Grade 3 & Grade 4 & Use of G-CFS \\
\hline Hematological & & & & \\
Leukopenia & $73(16.7 \%)$ & $8(1.8 \%)$ & $26(6 \%)$ & \\
Neutropenia & $93(21.3 \%)$ & $12(2.8 \%)$ & $41(9.4 \%)$ & $86(19.7 \%)$ \\
Non-hematological & & & - & \\
Allergic reaction & $21(4.8 \%)$ & - & - \\
Alopecia & $115(26.4 \%)$ & - & - \\
Anorexia & $25(5.7 \%)$ & - & - \\
Cough & $7(1.6 \%)$ & - & - \\
Diarrhea & $18(4.1 \%)$ & - & - \\
Fatigue/lethargy & $93(21.3 \%)$ & $7(1.6 \%)$ & - \\
Fever & $15(3.4 \%)$ & - & - \\
Hand-foot syndrome & $16(3.7 \%)$ & - & - \\
Mucositis & $41(9.4 \%)$ & $2(1.0 \%)$ & - \\
Peripheral neuropathy & $41(9.4 \%)$ & $2(0.5 \%)$ & - \\
Vomiting & $39(8.9 \%)$ & $1(0.2 \%)$ & - \\
Nausea & $50(11.5 \%)$ & - & - \\
Constipation & $4(0.9 \%)$ & - & - \\
Rash acneiform/skin rash & $18(4.1 \%)$ & - & - \\
\hline
\end{tabular}


Taiwan has provided reimbursement for use of eribulin by advanced breast cancer patients beginning in December 2014. Thus, prior to this date, patients received an anthracycline-based and taxane-based regimen. This is the reason we had so many patients who received eribulin, even after having received several prior treatments.

Compared with previous studies, we found that eribulin caused fewer and less severe AEs in Taiwanese women than in women treated in clinical trials $[9,10]$, but our $\mathrm{AE}$ results are comparable to the real-world experience reported by Iizumi et al. [17]. In particular, the rate of neutropenia was much lower in our population than reported for Westerners [9], and even for a Korean population [19] and for Japanese populations $[8,18]$. Although further studies are needed to identify the molecular, genetic, environmental, and socioeconomic factors that could explain these differences, one of the reasons for the better efficacy and the fewer and less severe AEs in our patients may be that the national health insurance system of Taiwan requires certification of the quality of care in all hospitals. Thus, this policy may have contributed to the improved care and survival of our cancer patients [20]. Another reason for the fewer and less severe AEs in our patients may be that almost all patients were restricted to $2 \mathrm{mg}$ as the highest dosage for every single injection, in an effort to control medical costs.

The major limitations of the present study are the retrospective and open label design, which could lead errors related to confounding or bias, and the use of a single treatment arm, with no comparators. Nonetheless, we examined a large population of Taiwanese women who received treatment of eribulin at 14 different hospitals in Taiwan, and used multivariable analysis to reduce the impact of confounding. The results of the present study of eribulin confirm that this drug is safe and effective when used to treat Taiwanese women with $\mathrm{LABC}$ or MBC who previously received at least 2 previous chemotherapy regimens that included an anthracycline and a taxane in either the adjuvant or the metastatic setting. Our data also confirm that eribulin maintains its favorable profile in terms of clinical effectiveness when used in daily clinical practice in heavily pretreated patients.

Acknowledgements This study is funded by Kaohsiung Breast Cancer Prevention and Education Society and Eisai Co., Ltd., Japan.

Disclosure All the authors declare that they have no conflicts of interest to disclose.

Open Access This article is distributed under the terms of the Creative Commons Attribution 4.0 International License (http://creativeco mmons.org/licenses/by/4.0/), which permits unrestricted use, distribution, and reproduction in any medium, provided you give appropriate credit to the original author(s) and the source, provide a link to the Creative Commons license, and indicate if changes were made.

\section{References}

1. Cardoso F, Harbeck N, Fallowfield L, Kyriakides S, Senkus E; ESMO Guidelines Working Group (2012) Locally recurrent or metastatic breast cancer: ESMO Clinical Practice Guidelines for diagnosis, treatment and follow-up. Ann Oncol 23(Suppl 7):vii11-vii19

2. d'Onofrio A, Mazzetta C, Robertson C, Smans M, Boyle P, Boniol M (2016) Maps and atlases of cancer mortality: a review of a useful tool to trigger new questions. Ecancermedicalscience 10:670

3. Colozza M, de Azambuja E, Personeni N, Lebrun F, Piccart MJ, Cardoso F (2007) Achievements in systemic therapies in the pregenomic era in metastatic breast cancer. Oncologist $12: 253-270$

4. Visconti R, Grieco D (2017) Fighting tubulin-targeting anticancer drug toxicity and resistance. Endocr Relat Cancer 24:T107-T117

5. Towle MJ, Salvato KA, Budrow J, Wels BF, Kuznetsov G, Aalfs KK, Welsh S, Zheng W, Seletsky BM, Palme MH, Habgood GJ, Singer LA, Dipietro LV, Wang Y, Chen JJ, Quincy DA, Davis A, Yoshimatsu K, Kishi Y, Yu MJ, Littlefield BA (2001) In vitro and in vivo anticancer activities of synthetic macrocyclic ketone analogues of halichondrin B. Cancer Res 61:1013-1021

6. Swami U, Chaudhary I, Ghalib MH, Goel S (2012) Eribulin-a review of preclinical and clinical studies. Crit Rev Oncol Hematol 81:163-184

7. Li J, Ren J, Sun W (2017) Systematic review of ixabepilone for treating metastatic breast cancer. Breast Cancer 24:171-179

8. Aogi K, Iwata H, Masuda N, Mukai H, Yoshida M, Rai Y, Taguchi K, Sasaki Y, Takashima S (2012) A phase II study of eribulin in Japanese patients with heavily pretreated metastatic breast cancer. Ann Oncol 23:1441-1448

9. Cortes J1, O'Shaughnessy J, Loesch D, Blum JL, Vahdat LT, Petrakova K, Chollet P, Manikas A, Diéras V, Delozier T, Vladimirov V, Cardoso F, Koh H, Bougnoux P, Dutcus CE, Seegobin S, Mir D, Meneses N, Wanders J, Twelves C; EMBRACE (Eisai Metastatic Breast Cancer Study Assessing Physician's Choice Versus E7389) investigators (2011) Eribulin monotherapy versus treatment of physician's choice in patients with metastatic breast cancer (EMBRACE): a phase 3 open-label randomised study. Lancet 377:914-923

10. Kaufman PA, Awada A, Twelves C, Yelle L, Perez EA, Velikova G, Olivo MS, He Y, Dutcus CE, Cortes J (2015) Phase III openlabel randomized study of eribulin mesylate versus capecitabine in patients with locally advanced or metastatic breast cancer previously treated with an anthracycline and a taxane. J Clin Oncol 33:594-601

11. Twelves C, Cortes J, Vahdat L, Olivo M, He Y, Kaufman PA, Awada A (2014) Efficacy of eribulin in women with metastatic breast cancer: a pooled analysis of two phase 3 studies. Breast Cancer Res Treat 148:553-561

12. Pivot X, Marmé F, Koenigsberg R, Guo M, Berrak E, Wolfer A (2016) Pooled analyses of eribulin in metastatic breast cancer patients with at least one prior chemotherapy. Ann Oncol 27:1525-1531

13. Watanabe J, Ito Y, Ohsumi S, Mizutani M, Tashiro H, Sakurai K, Takahashi M, Saito T, Tsurutani J, Mukai H, Yoshinami T, Takao S, Yamamoto Y, Matsuoka T, Iwase H, Iwata H, Nakamura S, Saeki T (2017) Safety and effectiveness of eribulin in Japanese patients with locally advanced or metastatic breast cancer: a postmarketing observational study. Invest New Drugs 35:791-799

14. Garrone O, Montemurro F, Saggia C, La Verde N, Vandone AM, Airoldi M, De Conciliis E, Donadio M, Lucio F, Polimeni MA, Oletti MV, Giacobino A, Merlano MC (2016) Eribulin in pretreated metastatic breast cancer patients: results of the TROTTER 
trial-a multicenter retrospective study of eribulin in real life. Springerplus 5:59

15. Watanabe $\mathbf{J}$ (2015) Eribulin monotherapy improved survivals in patients with ER-positive HER2-negative metastatic breast cancer in the real world: a single institutional review. Springerplus 4:625

16. Gamucci T, Michelotti A, Pizzuti L, Mentuccia L, Landucci E, Sperduti I, Di Lauro L, Fabi A, Tonini G, Sini V, Salesi N, Ferrarini I, Vaccaro A, Pavese I, Veltri E, Moscetti L, Marchetti P, Vici P (2014) Eribulin mesylate in pretreated breast cancer patients: a multicenter retrospective observational study. J Cancer $5: 320-327$

17. Iizumi S, Shimoi T, Tsushita N, Bun S, Shimomura A, Noguchi E, Kodaira M, Yunokawa M, Yonemori K, Shimizu C, Fujiwara Y, Tamura K (2017) Efficacy and safety of eribulin in patients with locally advanced or metastatic breast cancer not meeting trial eligibility criteria: a retrospective study. BMC Cancer 17:819
18. Maeda S, Saimura M, Minami S, Kurashita K, Nishimura R, Kai Y, Yano H, Mashino K, Mitsuyama S, Shimokawa M, Tamura K; Kyushu Breast Cancer Study Group (2017) Efficacy and safety of eribulin as first- to third-line treatment in patients with advanced or metastatic breast cancer previously treated with anthracyclines and taxanes. Breast 32:66-72

19. Park YH, Kim TY, Im YH, Lee KS, Park IH, Sohn J, Lee SH, Im SA, Kim JH, Kim SH, Lee SJ, Koh SJ, Lee KH, Choi YJ, Cho EK, Lee S, Kang SY, Seo JH, Kim SB, Jung KH (2017) Feasibility and efficacy of eribulin mesilate in korean patients with metastatic breast cancer: Korean multi-center phase IV clinical study results. Cancer Res Treat 49:423-429

20. Ou-Yang F, Hsu NC, Juan CH, Huang HI, Moi SH, Chen FM, Liu TW, Hou MF (2015) Breast cancer quality of care in Taiwan in relation to hospital volume: a population-based cohort study. Asia Pac J Clin Oncol 11:308-313

\section{Affiliations}

\section{Kun-Ming Rau ${ }^{1,12} \cdot$ Fu Ou-Yang ${ }^{2,12,13} \cdot$ Ta-Chung Chao $^{3} \cdot$ Yao-Lung Kuo ${ }^{4} \cdot$ Tsui-Fen Cheng $^{5} \cdot$ Tsu-Yi Chao $^{6}$. Dar-Ren Chen ${ }^{7} \cdot$ Yen-Dun Tzeng ${ }^{8,12}$ • Being-Whey Wang ${ }^{8,12} \cdot$ Chun-Yu Liu $^{3} \cdot$ Ming-Hung Hu ${ }^{9} \cdot$ Yin-Che Lu $^{10}$. Wei-Jen Ou ${ }^{11}$. Chin-Ho Kuo ${ }^{10}$. Chieh-Han Chuang ${ }^{2,12,13}$ • Jung-Yu Kan²,12,13 · Fang-Ming Chen 2, 12,13,14,15 . Ming-Feng $\mathrm{Hou}^{2,12,13,15,16}$}

Kun-Ming Rau

kmrau58@adm.cgmh.org.tw

Fu Ou-Yang

swfuon@kmu.edu.tw

1 Division of Hematology-Oncology, Kaohsiung Chang Gung Memorial Hospital, No. 123, Dapi Rd, Niaosong Dist., Kaohsiung 833, Taiwan (ROC)

2 Division of Breast Surgery, Department of Surgery, Kaohsiung Medical University Hospital, No. 100, Tzyou 1st Road, Kaohsiung 807, Taiwan (ROC)

3 Division of Medical Oncology, Taipei Veterans General Hospital, No. 201, Sec. 2, Shipai Rd, Beitou District, Taipei 11217, Taiwan (ROC)

4 Department of Surgery, National Cheng-Kung University Hospital, No. 138, Sheng Li Road, Tainan 704, Taiwan (ROC)

5 Breast Cancer Center, Shin Kong Wu Ho-Su Memorial Hospital, B3, No. 135, Wenchang Rd., Shilin Dist., Taipei 111, Taiwan (ROC)

6 Division of Hematology-Oncology, Taipei Medical University- Shuang Ho Hospital, No. 291, Zhongzheng Rd., Zhonghe District, New Taipei City 23561, Taiwan (ROC)

7 Department of Surgery, Changhua Christian Hospital, No. 135, Nanxiao St., Changhua 500, Taiwan (ROC)
8 Division of General Surgery, Kaohsiung Veterans General Hospital, No. 386, Dazhong 1st Rd., Zuoying Dist., Kaohsiung 813, Taiwan (ROC)

9 Division of Hematology and Oncology, Cardinal Tien Hospital, No. 362, Zhongzheng Rd., Xindian Dist., New Taipei City 231, Taiwan (ROC)

10 Division of Hematology-Oncology, Chia-Yi Christian Hospital, No. 539, Zhongxiao Rd., East Dist., Chiayi 60002, Taiwan (ROC)

11 Division of Medical Oncology, Landseed Hospital, No. 77, Guangtai Rd., Pingzhen Dist., Taoyuan 324, Taiwan (ROC)

12 Kaohsiung Breast Cancer Prevention and Education Society, 10B, No. 100, Tzyou 1st Road, Kaohsiung 807, Taiwan (ROC)

13 Department of Surgery, Kaohsiung Municipal Ta-Tung Hospital, No. 68, Zhonghua 3rd Rd., Qianjin Dist., Kaohsiung 801, Taiwan (ROC)

14 Cancer Center, Kaohsiung Municipal Ta-Tung Hospital, No. 68, Zhonghua 3rd Rd., Qianjin Dist., Kaohsiung 801, Taiwan (ROC)

15 Department of Surgery, Faculty of Medicine, College of Medicine, Kaohsiung Medical University, No. 100, Tzyou 1st Road, Kaohsiung 807, Taiwan (ROC)

16 Department of Surgery, Kaohsiung Municipal Hsiao-Gang Hospital, No. 482, Shanming Rd, Siaogang Dist., Kaohsiung 812, Taiwan (ROC) 